\title{
Preparation of function-enhanced vegetable oils
}

\author{
Hiroshi Maeda ${ }^{1}$, Takao Satoh ${ }^{2}$ and Waliul Md. Islam ${ }^{1,3}$ \\ ${ }^{1}$ Institute of Drug Delivery Science, Sojo University, Kumamoto, 860-0082, Japan; ${ }^{2}$ Kumamoto \\ Industrial Research Institute, Kumamoto, 862-0901, Japan; ${ }^{3}$ Department of Nano Science, Sojo \\ University, Kumamoto, 860-0082, Japan
}

Corresponding Author: Hiroshi Maeda, $\mathrm{PhD}, \mathrm{MD}$, Professor, Institute of Drug Delivery Science, Sojo University, 4-22-1 Ikeda, Nishi-ku, Kumamoto, 860-0082, Japan

Submission date: October 23, 2015, Acceptance date: January 15, 2016: Publication date: January 18, 2016

\begin{abstract}
:
Background: Highly purified commercial vegetable oils, such as rape seed/canola oil, corn oil, safflower oil and soybean oil have virtually no antioxidant activity against lipid peroxyl radical scavenging activity (ROO') (Figure 1), and peroxy nitrite (ONOO').
\end{abstract}

Methodology: Dried tomato-juice-waste-residues or dried wine-ferment-waste-residues were incubated with a highly purified oil obtained from the market, which is low-functional-grade oil (eg. canola oil), at room temperature for more than 2-3 weeks. Their antioxidant activities were then examined as described below

Results: Antioxidant lipid soluble components such as lycopene/carotenoids, flavonoids, and chlorophylls, originally present in tomato-juice-waste-residue, wine-ferment-waste-residues and dried spinach leaf, etc, were transferred to such pure yet low-functional-grade vegetable oils. Peroxide value, acid value, and TBARS (thiobarbituric acid reactive substances) measurement, as well as spectroscopic characterization, demonstrated that such treatment much improved the properties of the low-functional-grade oils; namely low-functional-grade oils became highfunctional-grade oils. The absorption spectrum of dried waste of tomato-juice-waste-residue treated canola oil indeed became much improved against various oil-deteriorating effects.

Conclusions: This method provides an easy procedure to convert low-functional-grade oils to highfunctional-grade oils, using various vegetable-waste-residues.

Keywords: antioxidant components, enrichment of edible oils, lipid peroxidation, dried vegetable-waste-residues.

\section{INTRODUCTION:}


Background: Previously, Maeda et al found that most commercially available edible oils, which were processed by hexane extraction followed by a number of purification steps, were extremely low in anti-peroxyl radical (ROO), or radical scavenging activity. This greatly contrasts respective virgin oils, as exemplified by extra-virgin olive oil or crude rape seed oil [1-4] (Figure 1). Therefore, such highly purified oils will became prooxidant and less desirable food components in terms of health oriented diet. Oxidized oils may eventually cause DNA cleavages, modification of proteins, RNA, and lipids, as well as cellular damage or promotion of inflammation and carcinogenesis at later time [5-9].

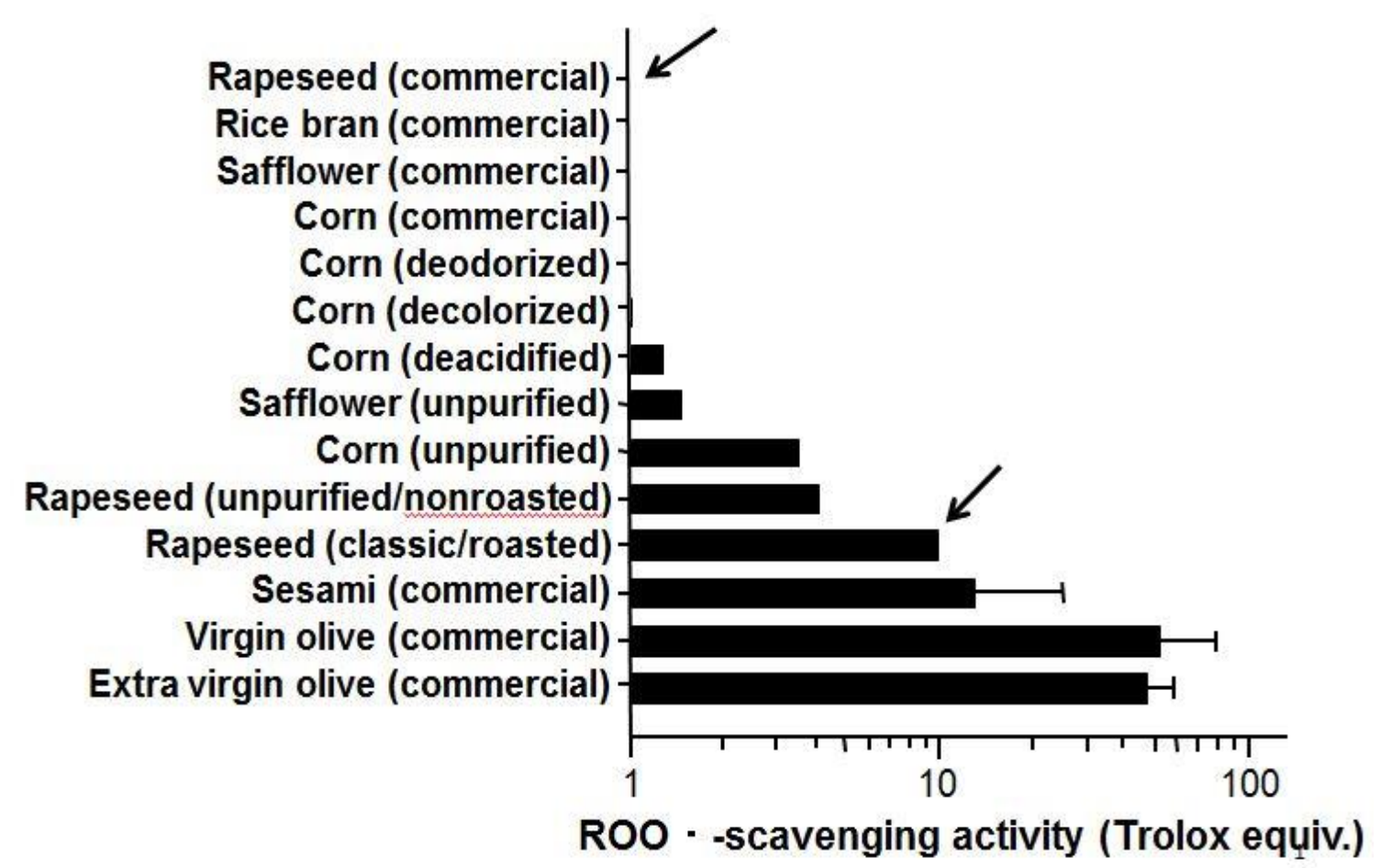

Figure 1: Peroxyl radical-scavenging activities of edible oils (Adapted from Ref. 4). With exception of extra virgin olive oils and unpurified or classic rape seed-and sesame-seed oils, most commercial highly purified oils are devoid of scavenging capacity against alkylperoxyl radicals (ROO).

These commercial oils of low antioxidant activity may be improved by adding functionally effective antioxidant components, by using dried vegetable-waste such as tomato-juice-wasteresidues and wine-ferment-waste-residues. Their antioxidant components will be transferred into the functionally poor grade edible oils, and consequently, one can improve the quality of such functionally poor oils and thereby contributing human health $[2,8,9]$. The purpose of this paper is to report a practical procedure to fortify low-functional-grade conventional edible oils to functionally enriched edible oils using dried vegetable-waste-residues such as tomato-juicewaste- residues, and wine-ferment-waste-residues, or other vegetable-waste-residues.

\section{METHODS:}

(1) Preparation and measurements of lycopene and carotenoid enriched oils. $100 \mathrm{ml}$ of commercial rape seed (canola) oil, $5.0 \mathrm{~g}$ or $1.0 \mathrm{~g}$ of the dried residue of tomato-juicewaste was added respectively. Each mixture was incubated at room temperature in dark 
for several weeks. Amount of lycopene and carotenoids extracted into the oil was monitored by increase of absorption $(400-550 \mathrm{~nm}$ ) and fluorescence at $470 \mathrm{~nm}$ of carotenoid. Wine-ferment-waste-residue was similarly prepared after hot air drying, and immersed in canola oil.

(2) Evaluation of function-enriched edible oils: Preventive effect of lipid peroxidation;

(a) Heat exposure: Commercial rape-seed oil and extra virgin oil were used as controls. Preparation of the test samples (function fortified oils) of tomato-juice-waste-residue and grape wine-ferment-waste-residues are described in the section above (1). All oil preparations were exposed to high temperature at $150^{\circ} \mathrm{C}$ and peroxide value (POV), acid value and TBARS were measured at various times as described in Figures 3 and 4. The methods of measurements of POV, acid value and TBARS were adapted conventional standard method described elsewhere [10-12]. The methods for the determination of POV (peroxide value) and AV (acid value) were based on the standard methods of Japan Oil Chemists' Society [12].

(b) Light exposure: Similar to (a) they were exposed to excessive light using Nippon Ikaga Kikai (Tokyo), LH-200-RDS equipped with fluorescent light tube (x3), NEC FL40S-2XN, 3.2 K lux at 390-730 nm.

\section{RESULT AND DISCUSSIONS:}

(1) Extraction of carotenoids etc.

Modern edible oil refining processes are highly elaborated and efficient. Most commercially available edible oils in the market are so purified that many important antioxidant components are mostly removed during refining process. Oils became primarily colorless and odorless; and they are now devoid of antioxidant or peroxyl radical scavenging components. We measured anti-alkyl peroxyl radical (ROO') scavenging activity, and found that many commercial oils have very little such activity [1-4] (Figure 1).

There is a significant disposal of tomato juice or extraction waste-residues in wine making which still contain significant amounts of functionally useful components that may be recovered by immersing the dried waste residues in the functionally poor oil, ie., low grade oils. Figure 2A, $\mathrm{B}$ shows it was indeed possible to recover such component like lycopene and carotenoids, and the spectrum of this oil exhibits multiple peaks correspond tomato lycopene. See also Figure 5.

(2) Antioxidant activity:

(a) Acid value after light and heat exposure. Commercial highly purified edible oils are vulnerable for oxidation and resulting in lipid or alkyl hydroperoxides formation (ROOH), which undergo formation lipid (alkyl) peroxyl radicals which (ROO') in the presence of heme or other metallic compounds $(\mathrm{ROOH} \rightarrow \mathrm{ROO}$ ) (Figure 1) [5, 11]. This peroxyl radical can break DNA/RNA or damage proteins and lead to cell death [4-7, 11].

Time course of carotenoids extraction into the purified canola oil, in which 1 or 5\% (dry $\mathrm{wt} / \mathrm{wt}$ ) of tomato-juice-waste-residue yielded a significantly high carotenoid values, and extraction reached a plateau in about 13 to 21 days (Fig. 2A). The absorption spectrum shows multiple peaks corresponding lycopene (Fig. 2B). 

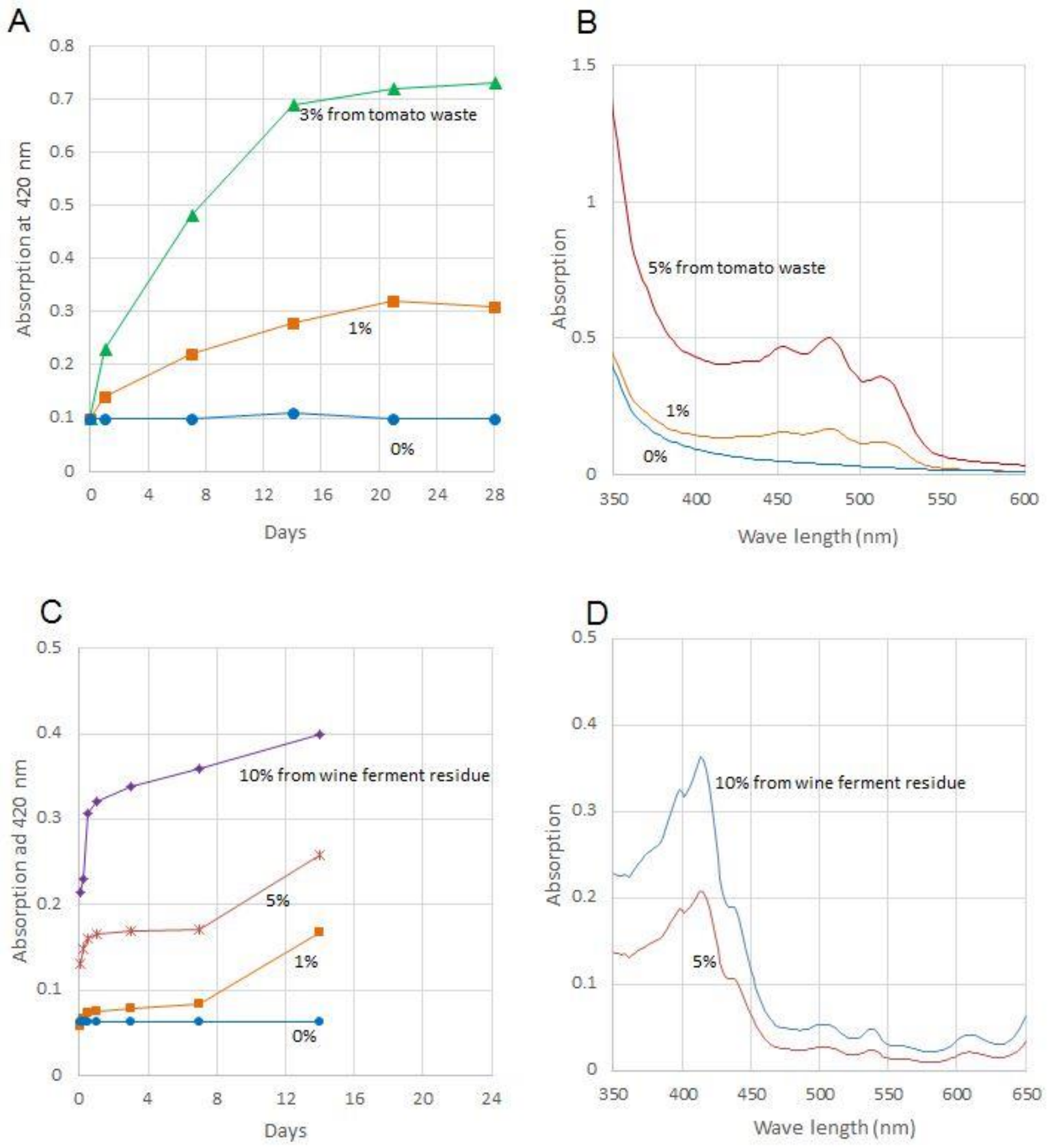

Figure 2: Transfer of lipid soluble functional components from dried tomato-juice-waste-residue as monitored by $420 \mathrm{~nm}$ (A). After preparation of tomato juice, the waste-residue was air dried and used as a material for extraction purpose. $-\bullet-$, no tomato residue, control canola oil; $-\square-, 1 \%$ of dried waste-residue of tomato juice; $-\boldsymbol{\Delta}-, 5 \%$ waste-residue. Incubation was at room temperature $\left(25^{\circ} \mathrm{C}\right)$. After 3 weeks incubation for still-extraction it reach a plateau, ie, extraction was almost completed. Increase of absorption during incubation reflecting extracted lycopene and carotenoids or alike. (B) shows absorption spectra; multiple peaks between 420-550 $\mathrm{nm}$ reflecting that of lycopene. An absorption spectra were taken for the sample on 21 days, respectively. (C) and (D) are similar to (A) and (B) above obtained for wine-ferment-waste-residue. Absorption spectra were taken on 14 days incubation. 
(b) Anti-POV after heat and light exposure. Upon exposure to oxidation condition, POV was significantly suppressed to $25 \%$ after $5 \mathrm{hrs}$ at $150^{\circ} \mathrm{C}$ and light exposure (above) to the $5 \%$ (wt/wt) tomato-juice-waste-residue treated oil, and showed suppression of POV to about $25 \%$ (net) at 5 hrs (Fig. 3).
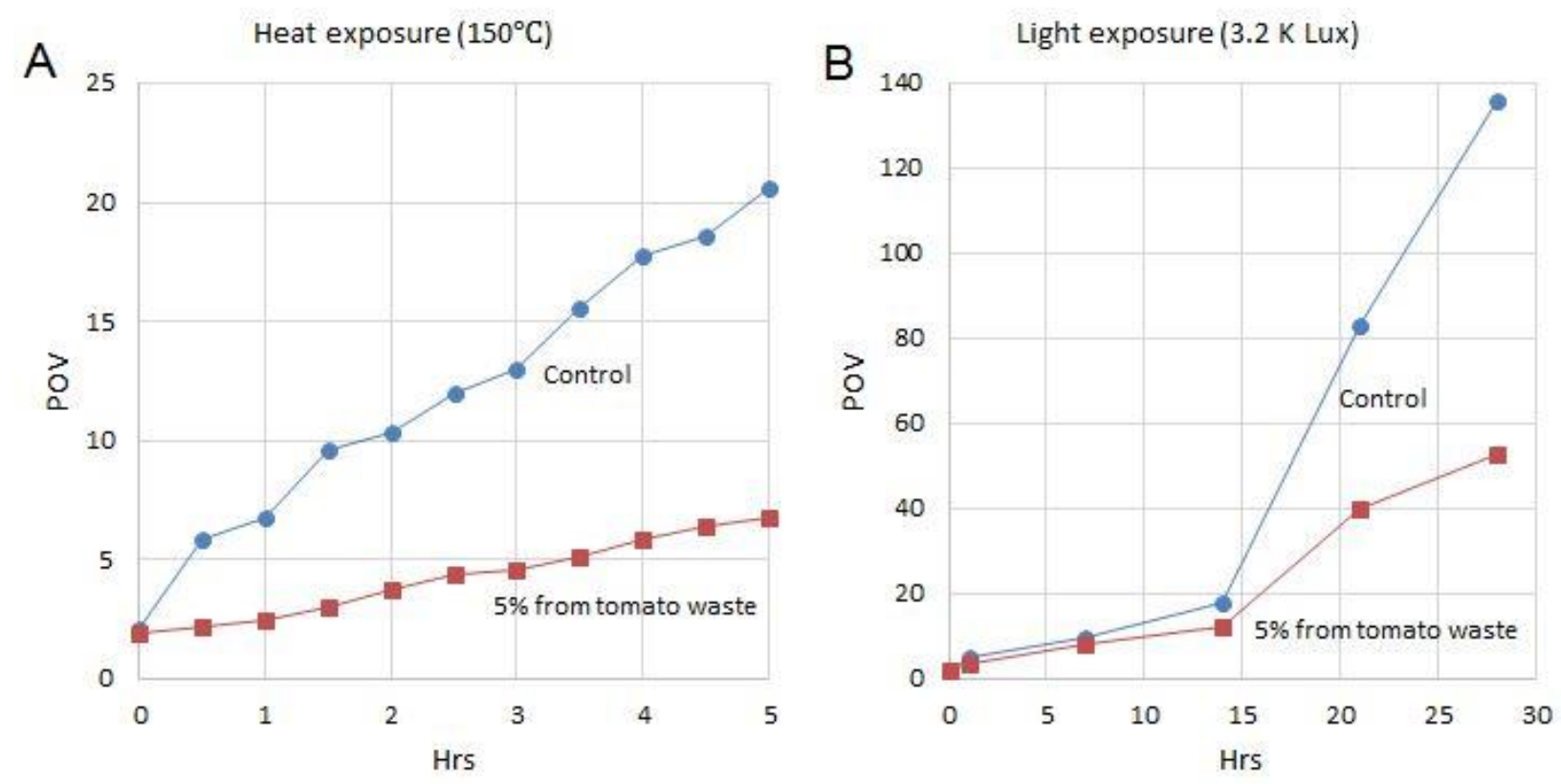

Figure 3: Suppressive effect on heat and light induced oil oxidation. (A) Peroxide value (POV) was measured by a standard method. After $5 \mathrm{hrs}$ incubation at $150^{\circ} \mathrm{C}$, tomato-juice-waste-residue treated canola oil showed about net $75 \%$ suppression of POV to the control canola oil. (B) Light induced oxidation was carried out under artificial climate unit NEC model, LH-200-RDS with fluorescent light tubes FL-40S, light wavelength was $390-730 \mathrm{~nm} 3.2 \mathrm{~K}$ lux. This also shows suppression of POV in the tomato-juice-waste-residue treated oil. It became significant after $20 \mathrm{hrs}$ or longer time of irradiation.

(c) TBARS value after light and heat exposure. Figure 4C, D shows results of function fortified effect in oils treated with tomato- and wine-ferment-waste-residues. They also showed significant suppression against the increase of acid value and POV (Figure 4A, B), which is consistent with absorption spectra.

These results indicate antioxidant components in dried tomato-juice-waste-residues and wine-ferment-waste-residues were extracted into commercial low-functional-grade rapeseed oil (see Figure 2A-D). As a result transferred carotenoid color is clearly visible as shown in Figure 5. The results warrant a simple procedure to convert low quality edible oils to function-enriched high grade oils. This procedure not only prevents oxidation of oils, but is also beneficial in providing various functional components such as polyphenolics, flavonoids, carotenoid, or lycopene. Therefore, this method and the products obtained will ultimately benefit human health, such as in the prevention of cancer and inflammation $[8,9,11]$.

We have previously established that alkyl peroxyl radical facilitates the promotion step in carcinogenesis, and that this was suppressed by various vegetable soup extracts, thereby suppressing promotion step in multistep carcinogenesis $[2,4,13]$. The step may be related to the suppression of inflammatory process as well via activation of cyclooxygenase I and i-NOS, as well as tumor necrosis factor $[8,9]$. 

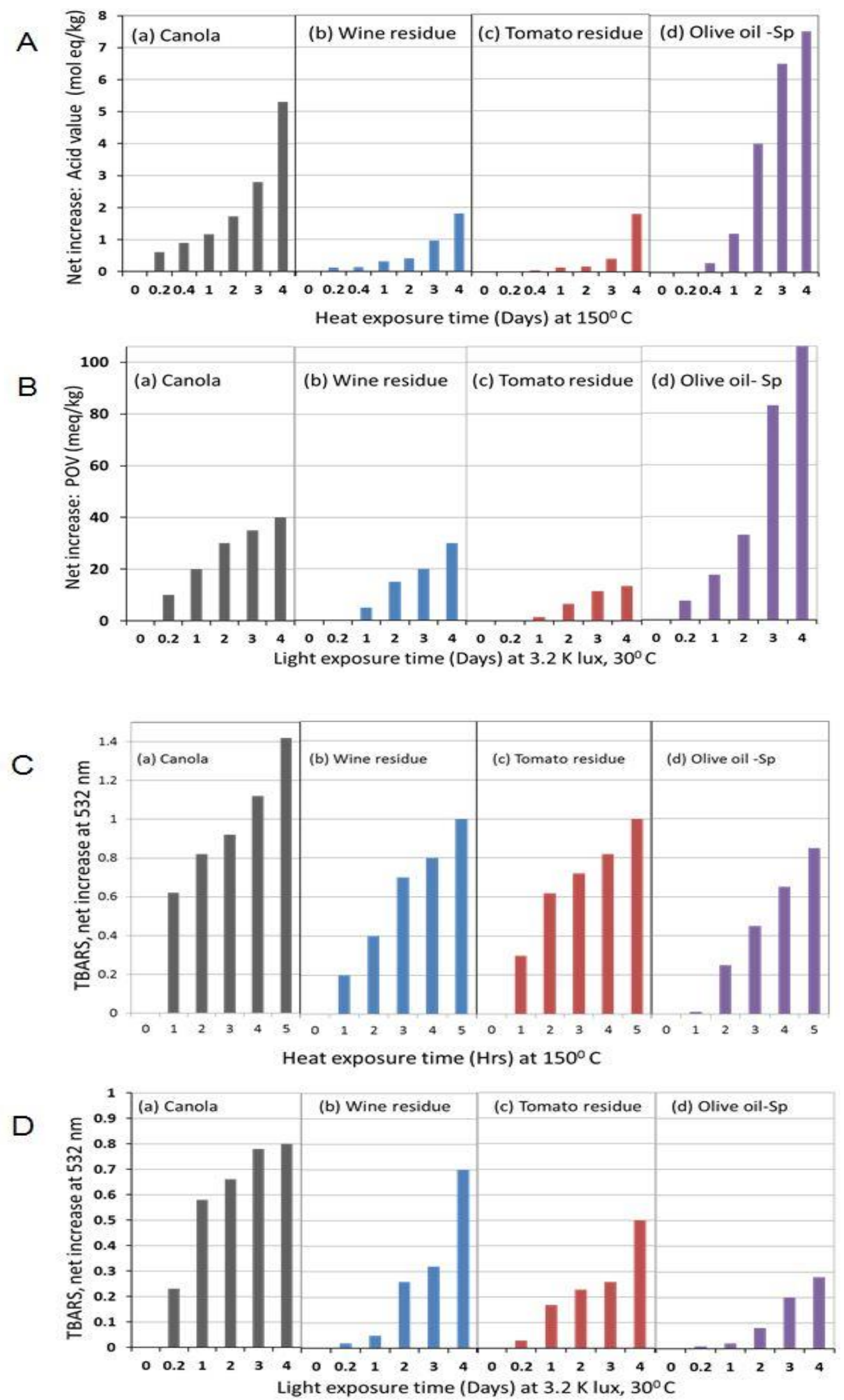

Figure 4: Effect of heat induced air oxidation on acid value (A), and light exposure on POV (B). In (A) heat treatment was carried out under ambient light at $150^{\circ} \mathrm{C}$ upto four days. Test samples, were control canola oil (a), wine ferment waste-residue (b) and 5\% tomato-juice-waste-residue treated canola 
oil (c) and extra virgin olive oil from Spain (d) were compared. Both acid value (A) and POV value (B) showed significant net suppression compared with control canola oil: the data indicate functional enrichment in the tomato-juice-waste-residue as well as wine ferment-waste-residue treated oils. (C) and (D) shows results of TBARS on the functionally fortified canola oil by heat and light exposure, respectively. (a) Control is the original canola oil without my treatment, (b) wine ferment-waste-residue, (c) tomato-juice-waste-residue and (d) extra virgin olive oil (from Spain).

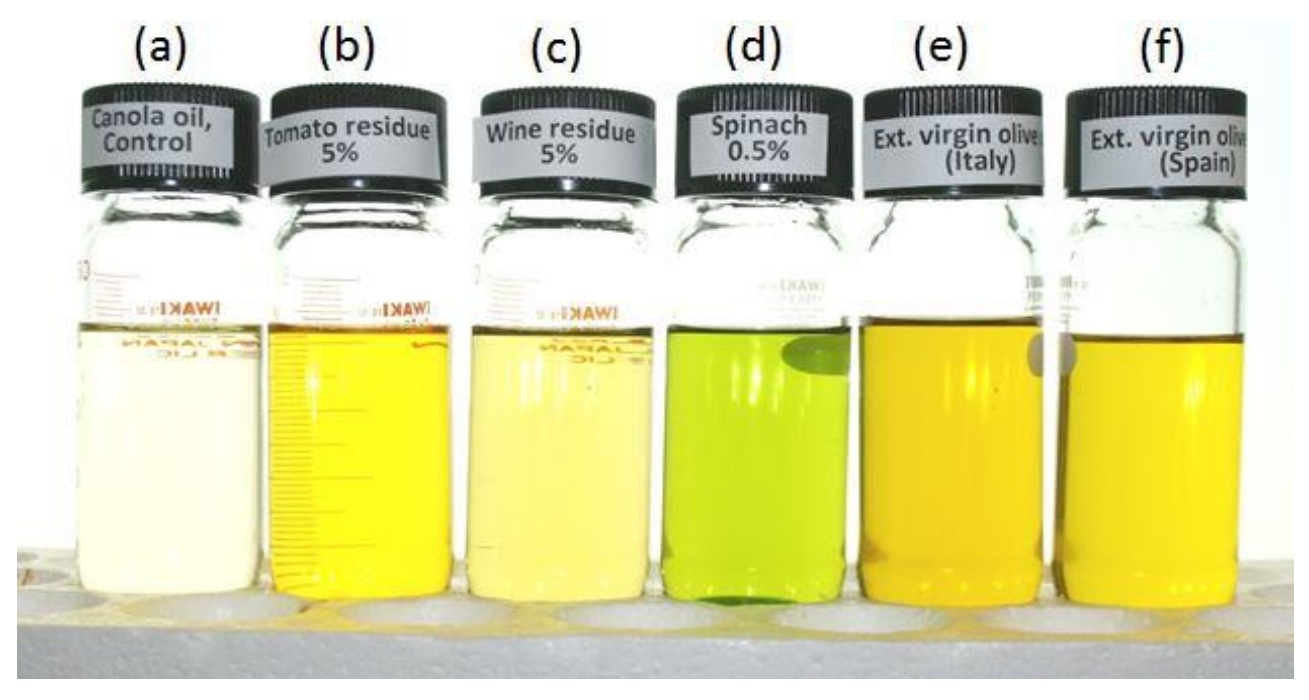

Figure 5: Comparison of visual observation of (a) control canola oil, (b) $5 \%$ dry wt/wt tomato-juice-waste-residue treated oil, (c) 5\% grape-ferment-waste-residues treated, (d) $0.5 \%$ dried spinach leaf treated oil, and (e) and (f) are extra virgin olive oils of Italy and Spain, respectively

In the manufacturing of traditional Japanese rape-seed (canola) oil, the seeds were roasted at high temperature. We found during this heating process, new antioxidant component (canolol), which is a potent scavenger of alkylperoxyl radical and peroxinitrite (ONOO`), was efficiently generated, and the oil increased in health benefits, especially when compared to the highly purified rape seed oil in the market, which are prepared by hexane extraction and other purification steps.

In the past few decades, vegetable oils have not been considered healthy dietary components. This is probably due to important antioxidant components, which originally existed as intrinsic components in the seeds, which unfortunately were removed during manufacturing and purification processes. As a result, vegetable oils became prooxidant or procarcinogen, which thereby led to its categorization as an unhealthy food product.

\section{CONCLUSIONS:}

Our method described in the article demonstrates an easy enrichment procedure for functional components of edible oils utilizing wasted-residues of vegetable including tomato-juice-waste residues and wine-ferment-waste residues. We highly recommend this process to further research and development of healthier food products in the future. 
Competing Interests: The authors have no financial interests or any other conflicts of interest to disclose.

Author's Contributions: All authors contributed to this study. HM designed, laid out experimental procedures, and wrote the manuscript. TS carried out extraction of tomato- and wine-ferment-waste-residues, and spectroscopy, WI carried out experiment of POV, AV and TBARS.

Acknowledgments: HM is grateful for a research award grant from Japan Health Foundation, 2015

\section{REFERENCES:}

1. Kanazawa A, Sawa T, Akaike T, Maeda H: Generation of lipid peroxyl radicals from oxidized edible oils and heme-iron. In Free Radicals in Food: Chemistry, Nutrition and Health Effects. Eds. by Morello MJ, Shahidi F, Ho CT. ACS Symposium Series; 807, Washington, D.C: Am. Chem. Soc.; 2002:282-300.

2. Sawa T, Akaike T, Kida K, Fukushima Y, Takagi K, Maeda H: Lipid peroxyl radicals from oxidized oils and heme-iron: Implication of a high-fat diet in colon carcinogenesis. Cancer Epidemiol Biomark Prevent 1998, 7:1007-1012.

3. Kanazawa A, Sawa T, Akaike T, Morimura S, Kida K, and Maeda H: Generation of lipid peroxyl radicals from edible oils and their biological activities: A need for consideration for anti-radical components and purification processing. BioFactors 2000, 13:187-193.

4. Kanazawa A, Sawa T, Akaike T, and Maeda H: Dietary lipid peroxidatin products and DNA damage in colon carcinogenesis. Eur J Lipid Sci Tech 2002, 104:439-447.

5. Kanazawa A, Sawa T, Akaike A, Maeda H: Formation of abasic sites in DNA by t-butyl peroxyl radicals: implication for potent genotoxicity of lipid peroxyl radicals. Cancer Letters 2000, 156:51-55.

6. Kuwahara H, Kanazawa A, Wakamatsu D, Morimura S, Kida K, Akaike T, Maeda H: Antioxidative and antimutagenic activities of 4-vinyl-2, 6-dimethoxyphenol (canolol) isolated from canola oil. J Agric Fd Chem 2004, 52:4380-4387.

7. Kuwahara H, Kariu T, Fan J, Maeda H: Generation of drug-resistant mutants of Helicobacter pylori in the presence of peroxynitrite, a derivative of nitric oxide at pathophysiological concentration. Microbiol Immunol 2009, 53:1-7.

8. Cao X, Tsukamoto T, Seki T, Tanaka H, Morimura S, Cao L, Mizoshita T, Ban H, Toyoda T, Maeda H, Tatematsu M: 4-Vinyl-2,6-dimethoxyphenol (canolol) suppresses oxidative stress and gastric carcinogenesis in Helicobacter pylori-infected carcinogen-treated Mongolian gerbils. Intl J Cancer 2008, 122:1445-1454.

9. Fang J, Seki T, Tsukamoto T, Qin H, Yin H, Liao L, Nakamura H, Maeda H: Protection from inflammatory bowel disease and colitis-associated carcinogenesis with 4-vinyl-2,6dimethoxyphenol (canolol) involves suppression of oxidative stress and inflammatory cytokines, Carcinogenesis 2013, 34:2833-2841. 
10. Akaike T, Sato K, Ijiri S, Miyamoto Y, Kohno M, Ando M, Maeda H: Bactericidal activity of alkyl peroxyl radicals generated by heme-iron-catalyzed decomposition of organic peroxides. Arch Biochem Biophys 1992, 294:55-63.

11. Sawa T, Nakao M, Akaike T, Ono K, Maeda H: Alkylperoxyl radical-scavenging activity of various flavonoids and other phenolic compounds: Implications for the anti-tumor-promoter effect of vegetables. J Agric Fd Chem 1999, 47:397-402.

12. Anonymous: (a) JOCS standard methods for the fat, oil and related materials. Manual of Japan Oil Chemists' Society 2013: 2.5.2.1-2013.

(b) JOCS standard methods for the fat, oil and related materials. Manual of Japan Oil Chemists' Society 2013: 2.3.1-2013.

13. Maeda H, Katsuki T, Akaike T, Yasutake R: High correlation between lipid peroxide radical and tumor-promoter effect: Suppression of tumor promotion in the Epstein-Barr virus/Blymphocyte system and scavenging of alkyl peroxide radicals by various vegetable extracts. Jpn J Cancer Res 1992, 83:923-928. 